\title{
Impact of pharmacy channel on adherence to oral oncolytics
}

\author{
Michael Stokes ${ }^{*}$ D , Carolina Reyes ${ }^{2}$, Yu Xia ${ }^{3}$, Veronica Alas ${ }^{4}$, Hans-Peter Goertz ${ }^{2}$ and Luke Boulanger ${ }^{5}$
}

\begin{abstract}
Background: Oral chemotherapy is increasingly prescribed to treat cancer. Despite its benefits, concerns have been raised regarding adherence to therapy. The study objective was to compare and measure adherence, persistence, and abandonment in patients filling prescriptions in traditional retail (TR) versus specialty pharmacy (SP) channels.

Methods: Using a retrospective cohort design, we selected newly treated patients aged $\geq 18$ years with a prescription for erlotinib, capecitabine, or imatinib during 2007-2011 from a Medco population of both United States commercial and Medicare health plans. Patients were classified according to pharmacy channel providing the medication. Abandonment was defined as a reversal following initial approval of the index prescription claim with no additional paid claims for agent within 90 days of reversal. Patients were considered adherent if the proportion of days covered between the date of the first and last oral prescription was $\geq 80 \%$.

Results: In our retrospective cohort, 11,972 filled their prescriptions within the SP channel, and 30,394 filled their prescriptions within the TR channels, respectively. The SP channel had the highest proportion of adherent patients compared with TR $(71.6 \%$ vs. $56.4 \%, P<.001)$. Abandonment of the initial prescription was low with overall rates of only $1.7 \%$. In multivariate models controlling for demographic characteristics, index oncolytic, days of supply, and copay, SP channel (relative to TR) was significantly associated with lower rates of abandonment and increased adherence.
\end{abstract}

Conclusions: Pharmacy channel may be influential on abandonment and adherence. Lower rates of abandonment and higher rates of adherence were observed among SP patients versus TR.

Keywords: Oral oncolytics, Abandonment, Adherence, Discontinuation, Specialty pharmacy

\section{Background}

Oral drug therapy use for patients with cancer has increased sharply over the last few years and this trend is expected to increase over the next several decades [1]. Experts estimate that oral medications comprise 25-35\% of the total cancer drugs in development [2-4]. In 2016, a report from IMS concluded that approximately $40 \%$ of total U.S. drug expenditures on targeted therapies were for oral formulations, up from $26 \%$ in 2010 [5].

Oral therapy provides potential advantages to patients. Oral agents may have different side effect profiles than their intravenous (IV) counterparts and patients may tolerate them better [6]. Patients often prefer oral agents to IV therapy because they offer more convenience as

\footnotetext{
* Correspondence: michael.stokes@evidera.com

${ }^{1}$ Evidera, Montreal, QC, Canada

Full list of author information is available at the end of the article
}

treatment interferes less with work and social activities, eliminating the need to travel to and from infusion clinics $[7,8]$. As oncologists become more sensitive to patient preferences and quality of life, treatment options that offer more convenience and flexibility are likely to be used more often [9].

Despite the benefits of oral therapy, concerns have been raised regarding the risks of nonadherence as the responsibility of managing regimens and monitoring toxicities are centered more directly on the patient $[1,2,10,11]$. One concern is under-dosing as a result of either nonadherence or dose reductions [12]. Under-dosing may lower drug plasma levels, thereby increasing the risk of cancer relapse or progression [13, 14]. Nonadherence may even cause clinicians to incorrectly attribute a patient's poor response to an absence of drug activity, which may lead to unnecessary diagnostic tests, hospitalizations, and changes 
in the dose or regimen $[9,15]$. Nonadherence is associated with an increased use of medical services [16].

Prior research has produced important findings regarding factors which adversely affect adherence in patients receiving oral oncolytics, including a lack of understanding of proper treatment administration, older age, complex dosing regimens, increased drug costs, and side effects [17-19]. However, there is limited data concerning the benefits of customized therapy management programs and whether specialty pharmacies can play a key role by working with physicians to ensure adherence and persistence in cancer patients. The purpose of this study was to examine this issue using a sample of patients newly treated with common oral oncolytics including erlotinib, capecitabine, and imatinib from the Medco research database. Erlotinib is approved for advanced non-small cell lung cancer (NSCLC) and advanced pancreatic cancer. Imatinib is approved for adult cancer indications including Philadelphia positive $(\mathrm{Ph}$ +) chronic myeloid leukemia, $\mathrm{Ph}+$ acute lymphoblastic leukemia, and unresectable/metastatic gastrointestinal stromal tumors. Capecitabine is indicated for colorectal and metastatic breast cancers.

\section{Methods}

\section{Data source}

A retrospective cohort analysis using the Medco research database was conducted to estimate the impact of the pharmacy channel on adherence and persistence in patients receiving oral oncolytics. Data were collected by Medco Health Solutions, formerly a large pharmacy benefits manager in the United States (US). As of 2011, the database included pharmacy claims from US commercial and Medicare health plans for over 60 million covered lives. Dates of health plan eligibility and demographic information were obtained from the eligibility files. The pharmacy claims included information on generic and brand medication names, national drug code (NDC), prescription fill dates, pharmacy channel, claim status (paid/ reversed), as well as quantity and days supplied. Patient and provider information contained within the Medco database are de-identified, making it compliant with the Health Insurance Portability and Accountability Act privacy regulations.

\section{Patient eligibility criteria}

The study sample included patients $\geq 18$ years old with a prescription claim for erlotinib, imatinib, or capecitabine between July 1, 2007 and September 30, 2011. These agents were chosen because they are widely used to treat cancer and were commonly observed in the Medco database. Patients were classified into treatment groups (erlotinib, imatinib, or capecitabine) based on the first study oral oncolytic observed (i.e., the index oral oncolytic) during the study period. Only those without claims for the index oncolytic during the 6-month period prior to the index date (baseline period) were included to identify newly treated patients. The index date was defined as the fill date of the first oncolytic prescribed during the study period. Study measures were assessed over a variable follow-up period starting from the index date and ending at the date of disenrollment of pharmacy benefits or December 31, 2011 (last day of available data), whichever occurred earlier.

\section{Pharmacy channel groups}

Patients were categorized into groups based on the pharmacy channel where they filled their index oncolytic prescription-Specialty (SP) or Traditional Retail (TR). Within the SP channel, pharmacists and nurses provide various customized therapy management services to patients aimed at encouraging adherence and adverse event monitoring. The TR channel consisted of a network of nearly 60,000 participating pharmacies throughout the US. Both the TR and SP pharmacy channels allowed for prescriptions with a daily supply $>30$ days.

\section{Abandonment, adherence, and persistence study outcomes}

Abandonment occurs when a patient brings a prescription to the pharmacy and the claim is denied by the insurance provider either due to a Prior Authorization or Step Therapy requirement. Abandonment of the index prescription in this study was defined when the adjudication status of the first oncolytic prescription claim was reversed and there were no additional paid claims for the oncolytic within 90 days of the reversal. Patients were classified as having an "initial challenge but overcome" if their first fill for the oral oncolytic was reversed (because the claim was initially denied by their insurance) and they were able to fill a subsequent prescription (claim status = paid) for the same drug within 90 days of the initial reversal. Patients were classified as "approved without challenges" if the status of their index oncolytic was paid. Patients with missing data were classified as "unknown".

Adherence to the index oral oncolytic was measured as the total days supply of all prescription fills between the first and last fill (days supply was capped at last fill date) divided by the number of days between the first and last fill (e.g., proportion of days covered). Values $\geq 80 \%$ were used as the cutoff value for defining adherence $[20,21]$. This definition excluded patients who had one prescription fill or abandoned their index oncolytic prescription.

Persistence was defined as the duration of continuous use of the study drugs and allowed for brief gaps ( $<60$ days) between the run-out date (fill date plus days supplied) of the prescription claim and the subsequent 
refill. Discontinuation was defined as an interruption in therapy $\geq 60$ days between the run-out date (fill date plus days supplied) of the prescription claim and the subsequent refill. The persistence definition excluded patients who abandoned their index oncolytic. Pharmacy claims with status as reversed were excluded from the calculation of adherence and persistence; paid claims only were assessed. For instances where patients filled a prescription prior to the run-out date of their previous fill, we assumed patients would finish the current prescription before starting the refill.

\section{Data analyses}

Primary data analyses were stratified according to pharmacy channel and included patients receiving erlotinib, imatinib, or capecitabine. Baseline demographic characteristics, including age, gender, geographical region, index study year and copay amount were summarized using descriptive statistics. For each patient, copay (per 30 day supply) was calculated by summing the copay amounts on prescription claims from the study index date until the end of follow-up and then dividing by the number of 30 day periods of total daily prescription supply dispensed. Copay was inflated to 2011 US dollars using the consumer price index. Copay is a fixed amount plan members pay out-of-pocket when filling prescriptions; this amount does not include other payments such as deductibles or coinsurance. Comparisons of baseline characteristics, adherence, abandonment, and persistence were assessed using $t$-tests for continuous variables and chi-square tests for categorical variables. Analyses examining the relationships between pharmacy channel and adherence and persistence were conducted according to index oncolytic received. We also estimated adherence and persistence measures among a subset of erlotinib patients with a pancreatic or lung cancer diagnosis recorded in the medical claims and for a subset of capecitabine patients with a breast or colorectal cancer diagnosis. As medical claims were only available for a subset of patients included in the study, these analyses were performed to confirm overall results in patients with a cancer diagnosis indicated for treatment with one of the oncolytics examined. Small sample sizes precluded analyses in imatinib patients. Finally, we examined adherence and persistence in a subset of patients who did not switch pharmacy channels during follow-up.

Logistic regression models were used to assess the impact of pharmacy channel on abandonment of the initial oncolytic prescription (yes vs. no [approved without challenges, initial challenge but overcome, and unknown groups]) and adherence (proportion of days covered: $\geq 80 \%$ vs. $<80 \%$ ). The adherence (proportion of days covered) model included age, gender, geographic region of residence, pharmacy channel (SP vs. TR), index oncolytic, average days of supply, and copay as covariates. Days of supply was included in the adherence model to rule-out the possibility that differences in adherence between SP and TR groups was simply related to differences in prescription days of supply. Since prescriptions with longer days of supply were thought only to affect abandonment of the initial oncolytic through higher copays, days of supply was omitted from the abandonment model. Statistical significance was evaluated at alpha $=0.05$. SAS, version 9.2 (SAS Institute Inc., Cary, NC) was used for all analyses.

\section{Results}

\section{Patient and pharmacy channel characteristics}

Table 1 presents baseline characteristics results stratified by pharmacy channel where patients filled their index oncolytic. From an initial sample of 46,533 patients with a prescription for erlotinib, capecitabine or imatinib during July 1, 2007 through September 30, 2011, 42,366 were included in this study after exclusion criteria were applied (age $<18$ years as of index date or prescription for the index oncolytic in the 6-month period prior to index). Among those included, 11,972 (28.3\%) were included in the SP cohort and 30,394 (71.7\%) in the TR cohort. SP patients were slightly younger compared with TR (mean age 63.9 vs. 64.4 years, $P<0.001$ ). There was a slightly higher proportion of males in SP channel compared with TR (45.2\% vs. $43.0 \%, P<0.001)$. The distribution of patients residing in different geographical regions was also different across pharmacy channels $(P<0.001)$. A higher proportion of SP patients resided in the northeast $(29.5 \%)$ versus TR (21.2\%). There was also significant variation in the frequency of patients receiving a particular index oncolytic across pharmacy channel. The average copay among SP patients was $\sim \$ 35$ lower than TR (\$221 vs. $\$ 256, P<0.001)$. Similar relationships were observed in the subsets of patients included in analyses of abandonment and persistence (data not shown).

Approximately $50 \%$ of the oral oncolytic prescriptions filled in the SP channel were classified as mail order and $50 \%$ were classified as brick-and-mortar retail locations. Seventy-nine percent of SP patients and $91 \%$ of TR filled prescriptions with average daily supplies $\leq 30$ days.

\section{Abandonment, adherence and persistence analyses}

Rates of abandonment of the initial prescription were higher among TR compared with SP (Table 2, 2.0\% vs. $0.8 \%, P<0.001)$. A higher proportion of SP patients versus TR also had their index oncolytic approved without challenges (Table $2,98.0 \%$ vs. $94.7 \%, P<0.001$ ).

A larger proportion of SP channel patients were more adherent (proportion of days covered $\geq 80 \%$ ) to their index oncolytic compared with TR $(71.6 \%$ vs. $56.4 \%$, $P<0.001$ ) (Table 2). The average proportion of days 
Table 1 Characteristics of patients according to pharmacy channel where index oral oncolytic prescription was filled ${ }^{a}$

\begin{tabular}{|c|c|c|c|c|c|}
\hline Characteristic $^{\mathrm{b}}$ & Specialty & & Traditional Retail & & $P$-value \\
\hline Number of Patients & 11,972 & & 30,394 & & \\
\hline Row Percent & $28.3 \%$ & & $71.7 \%$ & & \\
\hline Mean (SD) Age & $63.9(12.5)$ & & $64.4(12.9)$ & & $<0.001$ \\
\hline Age Group, N (\%): & & & & & $<0.001$ \\
\hline $18-34$ years & 178 & $1.5 \%$ & 493 & $1.6 \%$ & \\
\hline $35-44$ years & 620 & $5.2 \%$ & 1616 & $5.3 \%$ & \\
\hline $45-54$ years & 1895 & $15.8 \%$ & 4710 & $15.5 \%$ & \\
\hline $55-64$ years & 3452 & $28.8 \%$ & 8239 & $27.1 \%$ & \\
\hline $65-74$ years & 3032 & $25.3 \%$ & 7662 & $25.2 \%$ & \\
\hline $75-84$ years & 2625 & $21.9 \%$ & 6734 & $22.2 \%$ & \\
\hline $85+$ years & 170 & $1.4 \%$ & 940 & $3.1 \%$ & \\
\hline Male, N (\%): & 5416 & $45.2 \%$ & 13,068 & $43.0 \%$ & $<0.001$ \\
\hline Region, N (\%): & & & & & $<0.001$ \\
\hline Northeast & 3531 & $29.5 \%$ & 6439 & $21.2 \%$ & \\
\hline Midwest & 2054 & $17.2 \%$ & 6217 & $20.5 \%$ & \\
\hline South & 4173 & $34.9 \%$ & 10,960 & $36.1 \%$ & \\
\hline West & 1813 & $15.1 \%$ & 5107 & $16.8 \%$ & \\
\hline Unknown & 401 & $3.3 \%$ & 1671 & $5.5 \%$ & \\
\hline Index Study Year, N (\%) & & & & & $<0.001$ \\
\hline 2007 & 1118 & $9.3 \%$ & 2670 & $8.8 \%$ & \\
\hline 2008 & 2118 & $17.7 \%$ & 5880 & $19.4 \%$ & \\
\hline 2009 & 2860 & $23.9 \%$ & 8176 & $26.9 \%$ & \\
\hline 2010 & 3451 & $28.8 \%$ & 8240 & $27.1 \%$ & \\
\hline 2011 & 2425 & $20.3 \%$ & 5428 & $17.9 \%$ & \\
\hline Index Oncolytic Received, N (\%) & & & & & $<0.001$ \\
\hline Erlotinib & 4774 & $39.9 \%$ & 10,297 & $33.9 \%$ & \\
\hline Capecitabine & 4906 & $41.0 \%$ & 15,156 & $49.9 \%$ & \\
\hline Imatinib & 2292 & $19.1 \%$ & 4941 & $16.3 \%$ & \\
\hline Copay ${ }^{c}$ Amount Categorized & & & & & $<0.001$ \\
\hline$\$ 0-49$ & 7112 & $59.4 \%$ & 16,921 & $55.7 \%$ & \\
\hline$\$ 50-100$ & 1572 & $13.1 \%$ & 4127 & $13.6 \%$ & \\
\hline$\$ 101-150$ & 761 & $6.4 \%$ & 1877 & $6.2 \%$ & \\
\hline$\$ 151-200$ & 350 & $2.9 \%$ & 920 & $3.0 \%$ & \\
\hline$\$ 201-250$ & 257 & $2.2 \%$ & 656 & $2.2 \%$ & \\
\hline$\$ 251-350$ & 199 & $1.7 \%$ & 651 & $2.1 \%$ & \\
\hline$\$ 351-500$ & 268 & $2.2 \%$ & 900 & $3.0 \%$ & \\
\hline$\$ 501-1000$ & 694 & $5.8 \%$ & 2137 & $7.0 \%$ & \\
\hline$>\$ 1000$ & 759 & $6.3 \%$ & 2205 & $7.3 \%$ & \\
\hline Mean (SD) Copay ${ }^{\mathrm{C}}$ Amount (per 30 days) & \multicolumn{2}{|l|}{$220.7(548.2)$} & \multicolumn{2}{|l|}{$256.1(595.31)$} & $<0.001$ \\
\hline P25 & \multicolumn{2}{|l|}{16.4} & \multicolumn{2}{|l|}{15.0} & \\
\hline Median & \multicolumn{2}{|l|}{36.0} & \multicolumn{2}{|l|}{40.0} & \\
\hline P75 & \multicolumn{2}{|l|}{111.4} & \multicolumn{2}{|l|}{142.86} & \\
\hline Mean (SD) Days Supplied Per Prescription & \multicolumn{2}{|l|}{$33.7(20.7)$} & \multicolumn{2}{|l|}{$25.0(12.5)$} & $<0.001$ \\
\hline Median & \multicolumn{2}{|l|}{30.0} & \multicolumn{2}{|l|}{26.4} & \\
\hline
\end{tabular}

Note: Study index date refers to the first date that a patient attempted to fill the index oral oncolytic prescription.

Abbreviations: SD, standard deviation; N, number of patients; P25, 25th percentile; P75, 75th percentile

a Pharmacy channel defined based on the channel where patient filled their initial (index) oral oncolytic

${ }^{b}$ Group comparisons were made using 2 -sided Pearson chi-square for categorical measures, $t$-test statistic for mean age and Wilcoxon test for mean copay, $P$-values are presented for comparisons using traditional retail as reference group

'Mean copay amount (per 30 day supply) calculated using patients' prescription drug claims for index oral oncolytic from study index until end of follow-up and inflated to 2011 US dollars 
Table 2 Unadjusted measures of abandonment, adherence and persistence according to pharmacy channel

\begin{tabular}{|c|c|c|c|c|c|}
\hline Characteristic $^{a}$ & Specialty & & Traditional Retail & & $P$-value \\
\hline Number of Patients & 11,972 & & 30,394 & & \\
\hline Abandonment of Index Prescription & & & & & $<0.001$ \\
\hline Approved Without Challenges, N (\%) & 11,731 & $98.0 \%$ & 28,788 & $94.7 \%$ & \\
\hline Initial Challenge but Overcome, N (\%) & 118 & $1.0 \%$ & 875 & $2.9 \%$ & \\
\hline Initial Challenge Unknown if Overcome, $\mathrm{N}(\%)^{\mathrm{b}}$ & 3 & $0.0 \%$ & 43 & $0.1 \%$ & \\
\hline Abandoned, $\mathrm{N}(\%)^{c}$ & 97 & $0.8 \%$ & 604 & $2.0 \%$ & \\
\hline Unknown, $\mathrm{N}(\%)^{\mathrm{d}}$ & 23 & $0.2 \%$ & 84 & $0.3 \%$ & \\
\hline \multicolumn{6}{|c|}{ Adherence - Proportion of Days Covered between the First and Last Fill ${ }^{e}$} \\
\hline Mean (SD) & $0.86(0.2)$ & & $0.79(0.2)$ & & $<0.001$ \\
\hline Number with $>1$ fill & 9194 & & 22,219 & & \\
\hline Adherent, N (\%) & 6586 & $71.6 \%$ & 12,542 & $56.4 \%$ & $<0.001$ \\
\hline \multicolumn{5}{|c|}{ Adherence - Proportion Days Covered between First and Last Fill, N (\%) } & $<0.001$ \\
\hline $0-0.20$ & 12 & $0.1 \%$ & 246 & $1.1 \%$ & \\
\hline $0.21-0.40$ & 241 & $2.6 \%$ & 1273 & $5.7 \%$ & \\
\hline $0.41-0.60$ & 984 & $10.7 \%$ & 3815 & $17.2 \%$ & \\
\hline $0.61-0.70$ & 733 & $8.0 \%$ & 2571 & $11.6 \%$ & \\
\hline $0.70-0.79$ & 638 & $6.9 \%$ & 1772 & $8.0 \%$ & \\
\hline $0.80-0.90$ & 965 & $10.5 \%$ & 2182 & $9.8 \%$ & \\
\hline $0.90-1.00$ & 5621 & $61.1 \%$ & 10,360 & $46.6 \%$ & \\
\hline \multicolumn{6}{|c|}{ Persistence ${ }^{f, g}$ - Time until Discontinuation of Index Oncolytic (days) } \\
\hline Number Filling Prescription for Index Oncolytic & 11,849 & & 29,663 & & \\
\hline Mean (SD) & $186.2(235.2)$ & & $161.8(218.5)$ & & $<0.001$ \\
\hline Median & 94 & & 83 & & \\
\hline Minimum-Maximum & 0-1622 & & $0-1613$ & & \\
\hline
\end{tabular}

Abbreviations: $S D$ standard deviation, $N$ number of patients

${ }^{a}$ Group comparisons were made using 2-sided Pearson chi-square for categorical measures and $t$-test statistics for continuous measures, $P$-values are presented for comparisons using traditional retail as reference group

bunable to determine if hurdle was overcome because follow-up time was censored

'Abandonment measure refers to whether the patient was able to successfully fill prescription within 90 days following an initial challenge (if no, prescription was considered abandoned)

${ }^{d}$ No information provided regarding the patient's first fill status, could not determine if prescription was filled

${ }^{\mathrm{e}}$ Adherence definition excluded patients who only had one prescription fill or abandoned their index prescription (calculation included $n=9194$ and $n=22,219$ specialty and traditional retail patients, respectively)

${ }^{f}$ Time until discontinuation (in days) of index oral oncolytic, allowing for a 60-day gap in therapy between the run-out date of the medication and the subsequent fill

${ }^{9}$ The definition of persistence excluded patients who abandoned their index oncolytic (calculation included $n=11,849$ and $n=29,663$ specialty and traditional retail patients, respectively)

covered was also significantly higher for SP $(0.86)$ versus TR $(0.79, P<0.001)$ (Table 2$)$.

SP patients remained on their index oral oncolytic an average of 24.4 days longer versus TR (186.2 vs. 161.8 days, $P<0.001$ ) (Table 2 ).

Similar relationships were observed when the data were stratified according to index oncolytic received (Table 3) and in analyses examining the subset of patients with a cancer diagnosis recorded in the medical claims (Additional file 1) as well as the subset that did not switch pharmacy channels (Additional file 2). The results presented in Tables 2 and 3, Additional file 1, and Additional file 2 are not adjusted for differences in baseline characteristics.

\section{Multivariate analyses}

Table 4 presents results of the multivariate model for abandonment of the initial oncolytic prescription. After covariate adjustment, SP channel was associated with significantly lower rates of abandonment (odds ratio, OR [95\% confidence interval, CI], 0.44 [0.35-0.55]). Higher copay amounts were associated with a higher likelihood of abandonment.

Table 5 displays multivariate results for adherence (proportion of days covered $\geq 80 \%$ ) to the index oncolytic. After covariate adjustment, SP channel was associated with a higher level of adherence compared with TR (Odds ratio, OR [95\% CI], 1.99 [1.87-2.11]). Higher 
Table 3 Unadjusted measures of abandonment, adherence and persistence, by index oral oncolytic received and pharmacy channel

\begin{tabular}{|c|c|c|c|c|c|c|c|c|c|c|}
\hline \multirow[b]{2}{*}{ Characteristic $^{a}$} & \multicolumn{3}{|l|}{ Erlotinib } & \multicolumn{3}{|l|}{ Imatinib } & \multicolumn{4}{|c|}{ Capecitabine } \\
\hline & Specialty & \multicolumn{2}{|c|}{ Traditional Retail } & Specialty & \multicolumn{2}{|c|}{ Traditional Retail } & \multicolumn{2}{|c|}{ Specialty } & \multicolumn{2}{|c|}{ Traditional Retai } \\
\hline Number Prescribed Index Oncolytic & 4774 & \multicolumn{2}{|l|}{10,297} & 2292 & \multicolumn{2}{|c|}{4941} & \multicolumn{2}{|c|}{4906} & \multicolumn{2}{|c|}{15,156} \\
\hline Abandonment of Index Prescription & \multicolumn{3}{|l|}{$f$} & \multicolumn{3}{|l|}{ f } & \multicolumn{4}{|l|}{ f } \\
\hline Approved Without Challenges, N (\%) & $464397.3 \%$ & 9644 & $93.7 \%$ & $227199.1 \%$ & 4688 & $94.9 \%$ & 4817 & $98.2 \%$ & 14,456 & $95.4 \%$ \\
\hline Initial Challenge but Overcome, N (\%) & $66 \quad 1.4 \%$ & 358 & $3.5 \%$ & $7 \quad 0.3 \%$ & 157 & $3.2 \%$ & 45 & $0.9 \%$ & 360 & $2.4 \%$ \\
\hline Initial Challenge Unknown if Overcome, $\mathrm{N}(\%)^{\mathrm{b}}$ & $30.1 \%$ & 25 & $0.2 \%$ & $0 \quad 0.0 \%$ & 3 & $0.1 \%$ & 0 & $0.0 \%$ & 15 & $0.1 \%$ \\
\hline Abandoned, $\mathrm{N}(\%)^{\mathrm{c}}$ & $50 \quad 1.0 \%$ & 239 & $2.3 \%$ & $110.5 \%$ & 79 & $1.6 \%$ & 36 & $0.7 \%$ & 286 & $1.9 \%$ \\
\hline Unknown, N (\%) ${ }^{d}$ & $120.3 \%$ & 31 & $0.3 \%$ & $30.1 \%$ & 14 & $0.3 \%$ & 8 & $0.2 \%$ & 39 & $0.3 \%$ \\
\hline \multicolumn{11}{|c|}{ Adherence - Proportion of Days Covered between the First and Last Fill } \\
\hline MPR Mean (SD) & $92.1(14.0)^{f}$ & \multicolumn{2}{|c|}{$89.6(17.1)$} & $86.8(18.9)^{\mathrm{f}}$ & \multicolumn{2}{|c|}{$82.5(21.2)$} & \multicolumn{2}{|c|}{$80.7(20.4)^{f}$} & \multicolumn{2}{|c|}{$70.6(22.5)$} \\
\hline Number with $>1$ Fill & 3439 & \multicolumn{2}{|l|}{7091} & 2046 & \multicolumn{2}{|l|}{4230} & \multicolumn{2}{|l|}{3709} & \multicolumn{2}{|l|}{10,898} \\
\hline Adherent, N (\%) & $288884.0 \%{ }^{\mathrm{f}}$ & 5691 & $80.3 \%$ & $152774.6 \%{ }^{f}$ & 2813 & $66.5 \%$ & 2171 & $58.5 \%^{\mathrm{f}}$ & 4038 & $37.1 \%$ \\
\hline \multicolumn{11}{|c|}{ Persistence ${ }^{e}$ - Time until Discontinuation of Index Oncolytic (days) } \\
\hline $\begin{array}{l}\text { Number Filling Prescription for } \\
\text { Index Oncolytic }\end{array}$ & 4709 & \multicolumn{2}{|l|}{10,002} & 2278 & 4845 & & 4862 & & 14,816 & \\
\hline Mean (SD) & $146.0(166.7)^{f}$ & 135.8( & 69.9) & $423.2(359.0)^{f}$ & $394.5(3$ & 54.9) & 114.2 & $(120.2)^{f}$ & $103.2(1$ & 15.3) \\
\hline Median & 89 & 79 & & 324 & 292 & & 78 & & 63 & \\
\hline Minimum-Maximum & $0-1441$ & $0-1613$ & & $0-1622$ & $0-1601$ & & $0-131$ & & 0-1397 & \\
\hline
\end{tabular}

Note: NDC codes 50242006201, 50242006301, 50242006401, 54569584700, 54569584800, 54868529000, 54868544700, 54868547400 used to identify erlotinib, NDC codes $00004110020,00004110051,00004110116,00004110150,54569571700,54868414300-54868414303,54868526000-54868526009$ used to identify capecitabine, NDC codes 00078037366, 00078040105, 00078040134, 00078040215, 00078043815, 54569584600, 54868528900-54868528904, 54868542700-54868542703 used to identify imatinib

Abbreviations: $S D$ standard deviation, $N$ number of patients

${ }^{a}$ Group comparisons were made using 2-sided Pearson chi-square for categorical measures and $t$-test statistics for continuous measures within each index oncolytic population, $p$-values are presented for comparisons using traditional retail as reference group

bUnable to determine if hurdle overcome because data were censored

${ }^{\mathrm{c}}$ Abandonment measure refers to whether patient was able to successfully fill prescription within 90 days following an initial challenge (if no, prescription was considered abandoned)

${ }^{\mathrm{d}}$ No information provided regarding the patients first fill status, cannot determine if prescription was filled

eTime until discontinuation (in days) of index oral oncolytic, allowing for a 60 day gap in therapy between the run-out date of the medication and the subsequent fill

f $P<0.001$

copays were associated with lower adherence. The average prescription days of supply category $\geq 90$ days was associated with better adherence.

\section{Discussion}

We used data from the Medco research database to estimate the impact of pharmacy channel on adherence and persistence among patients receiving erlotinib, imatinib, or capecitabine during 2007-2011. Our results indicate that filling prescriptions in a SP channel may lead to greater adherence compared with TR. This finding is consistent with previous research examining SPs [22-25]. In addition, SP patients were less likely to abandon their initial prescription and remained on treatment longer versus TR. Prior studies have focused on adherence in cancer patients receiving oral therapies $[11,13$, 26-31]. However, there are limited data examining the impact of SP channel on adherence. The overall mean proportion of days covered (adherence) in the current study was $79 \%$ for the TR channel $(90 \%, 83 \%$, and $71 \%$ for TR patients receiving erlotinib, imatinib, and capecitabine, respectively) and $86 \%$ for SP $(92 \%, 87 \%$, and $81 \%$ for SP patients receiving erlotinib, imatinib, and capecitabine, respectively). Prior retrospective studies examining adherence to imatinib and utilizing prescription claims data have reported mean medication possession ratios between $73 \%-90 \%[28,29,31]$. Mean MPRs and adherence rates for imatinib estimated as part of this current study were within the reported range of these prior studies. Among studies of adherence to breast cancer medications utilizing prescription refills, adherence rates (proportion of days covered $\geq 80 \%$ ) over $1-2$ years varied significantly, from $62 \%-89 \%$ [32]. Many published rates in breast cancer are based on studies assessing adherence to tamoxifen regimens, which may differ significantly from typically shorter oral chemotherapy regimens $[26,32]$. A few studies assessing capecitabine adherence in patients with breast, colorectal, and GIST tumors were identified [11, 30, 33, 34]. One study was a randomized controlled trial of patients with breast cancer where 
Table 4 Logistic regression model: abandonment of initial oncolytic prescription

\begin{tabular}{|c|c|c|}
\hline Determinant & Odds Ratio & $95 \% \mathrm{Cl}$ \\
\hline \multicolumn{3}{|c|}{ Age, Reference $<65$ years } \\
\hline$\geq 65$ years & 1.25 & $1.04-1.51$ \\
\hline \multicolumn{3}{|c|}{ Gender, Reference Female } \\
\hline Male & 1.16 & $0.99-1.36$ \\
\hline \multicolumn{3}{|c|}{ Geographic Region, Reference South } \\
\hline Northeast & 0.64 & $0.51-0.81$ \\
\hline Midwest & 1.10 & $0.90-1.35$ \\
\hline West & 1.00 & $0.78-1.27$ \\
\hline Other/missing & 1.48 & $1.01-2.17$ \\
\hline \multicolumn{3}{|c|}{ Population, Reference Retail Pharmacy } \\
\hline Specialty & 0.44 & $0.35-0.55$ \\
\hline \multicolumn{3}{|c|}{ Copay, Reference \$0-49 } \\
\hline$\$ 50-100$ & 1.55 & $1.14-2.12$ \\
\hline$\$ 101-150$ & 1.55 & $1.02-2.35$ \\
\hline$\$ 151-200$ & 1.94 & $1.17-3.24$ \\
\hline$\$ 201-250$ & 3.43 & $2.15-5.47$ \\
\hline$\$ 251-350$ & 4.54 & $3.00-6.93$ \\
\hline$\$ 351-500$ & 5.22 & $3.65-7.46$ \\
\hline$\$ 501-1000$ & 4.89 & $3.71-6.44$ \\
\hline$>\$ 1000$ & 13.69 & $10.87-17.23$ \\
\hline \multicolumn{3}{|c|}{ Index Oncolytic, Reference Erlotinib } \\
\hline Capecitabine & 1.41 & $1.15-1.74$ \\
\hline Imatinib & 0.94 & $0.73-1.22$ \\
\hline
\end{tabular}

Abbreviations: $\mathrm{Cl}$ confidence interval

adherence was calculated as the number of doses taken (as measured by a microelectronic monitoring system) divided by the number of planned doses. Average adherence of $78 \%$ was reported across all cycles [34]. Other studies assessing capecitabine used patient self-reports and noted adherence rates of $76.7 \%$ [11] and 91\% [30] which were higher relative to the current study. A possible explanation for this is that Bhattacharya et al. and Winterhalder et al. relied on patient self-reports, which have a tendency to overstate compliance. In addition, the retrospective database design of the current study allowed for an observational period spanning multiple years whereas these prior studies examining capecitabine had follow-up periods of only 4-6 months [30]. The study by Walter et al., reported capecitabine adherence rates of only $61 \%$ with microelectronic monitoring system, which was more in line with the current study, compared to $99 \%$ with patient selfreport. Relatively few studies examining adherence to erlotinib were identified. One study from the Netherlands utilizing patient self-reports recorded a mean MPR of 96.8\% for erlotinib [35] which was comparable to the mean MPRs of $90 \%$ + reported in this study. Rates of
Table 5 Logistic regression model: adherence to index oral oncolytic $^{a}$

\begin{tabular}{|c|c|c|}
\hline Determinant & Odds Ratio & $95 \% \mathrm{Cl}$ \\
\hline \multicolumn{3}{|c|}{ Age, Reference $<65$ Years } \\
\hline$\geq 65$ years & 1.04 & $0.98-1.09$ \\
\hline \multicolumn{3}{|c|}{ Gender, Reference Female } \\
\hline Male & 1.15 & $1.09-1.21$ \\
\hline \multicolumn{3}{|c|}{ Geographic Region, Reference South } \\
\hline Northeast & 0.93 & $0.87-0.99$ \\
\hline Midwest & 1.12 & $1.04-1.20$ \\
\hline West & 0.97 & $0.90-1.05$ \\
\hline Unknown & 1.02 & $0.87-1.18$ \\
\hline \multicolumn{3}{|c|}{ Population, Reference Retail Pharmacy } \\
\hline Specialty & 1.99 & $1.87-2.11$ \\
\hline \multicolumn{3}{|c|}{ Copay, Reference \$0-49 } \\
\hline$\$ 50-100$ & 0.74 & $0.69-0.80$ \\
\hline$\$ 101-150$ & 0.83 & $0.75-0.92$ \\
\hline$\$ 151-200$ & 0.74 & $0.65-0.86$ \\
\hline$\$ 201-250$ & 0.65 & $0.55-0.77$ \\
\hline$\$ 251-350$ & 0.89 & $0.75-1.06$ \\
\hline$\$ 351-500$ & 0.93 & $0.80-1.09$ \\
\hline$\$ 501-1000$ & 0.92 & $0.83-1.02$ \\
\hline$>\$ 1000$ & 0.74 & $0.65-0.83$ \\
\hline \multicolumn{3}{|c|}{ Prescription Days of Supply, Reference $\leq 30$} \\
\hline $31-89$ & 0.28 & $0.26-0.30$ \\
\hline $90+$ & 1.66 & $1.35-2.03$ \\
\hline
\end{tabular}

Index Oncolytic, Reference Erlotinib

$\begin{array}{lll}\text { Capecitabine } & 0.16 & 0.15-0.17 \\ \text { Imatinib } & 0.53 & 0.49-0.58\end{array}$

Note: patients assumed to be adherent if proportion of days covered $\geq 80 \%$, Mean p[adherence] for SP $=0.76$, Mean p[adherence] for TR $=0.59$, Mean $\mathrm{p}$ [adherence] for patients receiving erlotinib $=0.83$, Mean p[adherence] for patients receiving capecitabine $=0.42$, Mean p[adherence] for patients receiving imatinib $=0.70$

Abbreviations: $\mathrm{Cl}$ confidence interval, $\mathrm{P}$ [adherence], predicted probability of adherence

${ }^{\mathrm{a}}$ Based on the proportion of days covered between first and last fill of index oncolytic, analysis includes patients who filled their index oncolytic $>1$ time as adherence could only be measured for these patients

adherence may vary across different studies due to factors such as study design, cancer type, patients' overall disease burden, or treatment received. As part of this study, we also performed analyses in a subset of erlotinib and capecitabine patients with a cancer diagnosis recorded in the medical claims. Among the erlotinib subset, $86 \%$ had lung cancer and $14 \%$ had pancreatic cancer. Forty-seven percent of the capecitabine subgroup had breast cancer and $53 \%$ had colorectal cancer. The mean proportion of days covered within both tumor and oral oncolytic subgroups were consistent with results of the full patient sample stratified only by oncolytic received. 
Our results suggest that customized therapy management programs instituted at SPs can have a positive influence on adherence and persistence in cancer patients prescribed oral oncolytics. SP therapy management programs provide additional services to patients which are likely to contribute to improved levels of adherence compared with TR. For example, when patients start new prescriptions in the SP channel, pharmacists or nurses counsel patients by telephone, often verifying that the appropriate dose has been prescribed, explaining adverse effects, potential drug interactions, dosing requirements, instructions for use, and storage requirements. Patients might also be contacted prior to each refill to monitor outcomes and encourage adherence. Some SPs even offer 24-h nursing support for patients throughout their therapy as well as home visits prior to dispensing the medication. Many SPs also have therapeutic centers dedicated to oncology with staff specializing in this area.

The level of cost sharing was also a significant factor in determining adherence to oral medication. Patients with higher copays were more likely to have lower rates of adherence and higher rates of abandonment of the index oncolytic. Many prior studies have demonstrated a clear relationship between higher copays and decreased adherence [36-38]. Other unmeasured factors such as total household income or the proportion of out-ofpocket expenses in relation to income may have also played a role in affecting adherence. These variables were not available in the database.

This study used data from a healthcare claims database. Claims data are primarily collected for reimbursement purposes rather than research. The classification of patients into study groups and the measures derived from the data relied on inferences based upon the information appearing on the claims. Thus, there is the potential for coding inaccuracies leading to the misclassification of certain events or measures of interest. For example, the abandonment measure was inferred from the information appearing on the claims. Claim reversals were examined as an indication that the patient was having difficulty filling the prescription. The accuracy of our algorithm in identifying true instances of abandonment is not known. It is possible that some reversed claims were excluded from our dataset if the reversal occurred prior to the claims being loaded into the database. Consequentially, the abandonment rate might have been underestimated. Indeed, a prior study using nationally representative pharmacy data reported first fill abandonment rates of $6.2 \%, 13.5 \%$, and $12.8 \%$ for capecitabine, imatinib, and erlotinib, respectively; rates which are higher compared to our study [39]. Finally, our adherence measure was not an actual measure of medication consumption. We assumed that patients actually took the medication after filling prescriptions.
There were differences observed in the baseline characteristics of patients filling prescriptions in the SP channel and those utilizing TR which could have influenced results. Therefore, multivariate analyses controlling for age, gender, geographic region of residence, index oncolytic, average days of supply, and copay were conducted to confirm unadjusted results showing that SP patients have higher rates of adherence (unadjusted $\mathrm{OR}=1.95$ ) and lower rates of abandonment (unadjusted $\mathrm{OR}=0.40)$ compared with TR. In multivariate models, SP channel compared with TR was associated with significantly lower rates of abandonment (adjusted OR $=0.44$ ) and higher rates of adherence (adjusted OR $=1.99$ ) affirming the unadjusted results.

Our analyses were also limited by the variables available in the database. Data on overall income was not available. Hence, we could not explore the burden of patients' outof-pocket expenses relative to their overall income, which could have had a greater effect on adherence compared with their actual expense. We did not know whether patients were receiving assistance with copays from patient programs which could have also influenced results. A minimum post-eligibility period was not used for analyses to avoid bias from excluding patients with shorter followup periods due to death. We assumed death was the most likely reason for health coverage termination and that bias due to censored data would be minimal. Mortality data were not available to confirm this assumption. For most patients, we did not have access to their medical claims data. Therefore, it was inferred that patients were taking their oral chemotherapy to treat advanced cancer. An analysis was conducted to assess patient subgroups with medical claims containing a cancer diagnosis indicated for treatment with an oncolytic. Subgroup analyses were consistent with overall results. We also assumed differences in comorbidity or the severity of the patients' cancer would be negligible across pharmacy channel cohorts and would not significantly influence abandonment or adherence metrics. Finally, it should be noted that some TR pharmacies also have adherence and specialty clinical programs; the degree to which these programs were implemented was not captured in the database. Therefore, it is difficult to determine what effect this may have had on results.

The rapid growth in the development of specialty pharmaceuticals including oral oncolytics has increasingly led to the transfer of responsibility for obtaining and administering complex and costly medications from healthcare providers and hospitals to patients in the community [40]. These changes have driven new models of care including the rise of customized therapy management programs with the aim of improving otherwise unfavorable medication use behaviors, mitigating waste, and improving outcomes [40]. The published evidence, 
although limited, does suggest that the majority of patient support and therapy management programs are having a positive impact [40].

\section{Conclusions}

Patients filling prescriptions in the SP channel were more likely to achieve adherence compared with patients using TR. Pharmacy channel also appears to be influential on abandonment, with lower rates observed among SP patients compared with TR.

\section{Additional files}

Additional file 1: Unadjusted measures of abandonment, adherence, and persistence: patient subset with cancer diagnosis code. (DOCX 15 kb)

Additional file 2: Unadjusted measures of abandonment, adherence, and persistence: patient subset who did not switch pharmacy channels. (DOCX $13 \mathrm{~kb})$

\section{Abbreviations}

IV: Intravenous; NDC: National drug code; NSCLC: Non-small cell lung cancer; Ph +: Philadelphia positive; SP: Specialty pharmacy; TR: Traditional retail; US: United States

\section{Acknowledgements}

The authors would like to acknowledge Kawthar Nakayima, a production assistant at Evidera who assisted with formatting and copy-editing of the manuscript.

\section{Funding}

Funding for this study was provided by Genentech.

\section{Availability of data and materials}

The data that support the findings of this study are available from Express Scripts but restrictions apply to the availability of these data, which were used under license for the current study, and so are not publicly available. Data are however available from the authors upon reasonable request and with permission of Express Scripts.

\section{Authors' contributions}

MS participated in the design, analysis and interpretation of the data, and the drafting of the manuscript. YX created the study analytic file from the raw data, carried out the statistical analysis, and participated in the revision of the manuscript. VA, LB, HPG, and CR participated in the design of the study, analysis and interpretation of the data, and revision of the manuscript. All authors read and approved the final manuscript.

\section{Competing interests}

MS is a full-time employee of Evidera. VA, YX, and LB were full-time employees of Evidera at the time the study was conducted. Evidera received funding from Genentech for the conduct of this study and drafting of the manuscript. Evidera is an independent consulting firm that was contacted by Genentech to perform this study. HPG and CR are full-time employees of Genentech and receive a salary from Genentech.

\section{Consent for publication}

Not applicable.

\section{Ethics approval and consent to participate}

Not Applicable; patient and provider information contained within the Medco database are de-identified, making it compliant with the Health Insurance Portability and Accountability Act privacy regulations. Permission to access the database was obtained from Medco Health Solutions, Inc.

\section{Publisher's Note}

Springer Nature remains neutral with regard to jurisdictional claims in published maps and institutional affiliations.

\section{Author details}

${ }^{1}$ Evidera, Montreal, QC, Canada. ${ }^{2}$ Genentech, San Francisco, CA, USA. ${ }^{3}$ Evidera, Lexington, MA, USA. ${ }^{4}$ GNS Healthcare, Cambridge, MA, USA. ${ }^{5}$ Truven Health Analytics, Cambridge, MA, USA.

Received: 1 December 2015 Accepted: 9 June 2017

Published online: 19 June 2017

\section{References}

1. Al-Barrak J, Cheung WY. Adherence to imatinib therapy in gastrointestinal stromal tumors and chronic myeloid leukemia. Support Care Cancer. 2013; 21(8):2351-7.

2. Weingart SN, Brown E, Bach PB, Eng K, Johnson SA, Kuzel TM, et al. NCCN task force report: oral chemotherapy. J Natl Compr Cancer Netw. 2008; 6(Suppl 3):S1-14.

3. D'Amato SL. New oral chemotherapeutic agents: part B vs. part D implications. In: Association of Community Cancer Centers; 2008.

4. Geynisman DM, Wickersham KE. Adherence to targeted oral anticancer medications. Discov Med. 2013;15(83):231-41.

5. IMS Institute for Healthcare Informatics. Developments in cancer treatment market dynamics, patient access and value: global oncology trend report 2015. In: QuintilesIMS Inc; 2015.

6. Carney DN. The pharmacology of intravenous and oral etoposide. Cancer. 1991;67(1 Suppl):299-302.

7. Borner M, Scheithauer W, Twelves C, Maroun J, Wilke H. Answering patients needs: oral alternatives to intravenous therapy. Oncologist. 2001;6(Suppl 4): 12-6.

8. Pelusi J. Capecitabine versus 5-FU in metastatic colorectal cancer: considerations for treatment decision-making. Community Oncol. 2006;3(1): 19-27.

9. Partridge $\mathrm{AH}$, Avorn J, Wang PS, Winer EP. Adherence to therapy with oral antineoplastic agents. J Natl Cancer Inst. 2002;94(9):652-61.

10. Hede K. Increase in oral cancer drugs raises thorny issues for oncology practices. J Natl Cancer Inst. 2009;101(22):1534-6.

11. Bhattacharya D, Easthall C, Willoughby KA, Small M, Watson S. Capecitabine non-adherence: exploration of magnitude, nature and contributing factors. $J$ Oncol Pharm Pract. 2012;18(3):333-42.

12. Barthelemy P, Asmane-De la Porte I, Meyer N, Duclos B, Serra S, Dourthe $L M$, et al. Adherence and patients' attitudes to oral anticancer drugs: a prospective series of 201 patients focusing on targeted therapies. Oncology. 2015;88(1):1-8.

13. Tsang J, Rudychev I, Pescatore S. Prescription compliance and persistency in chronic myelogenous leukemia and gastrointestinal stromal tumor patients on imatinib. 2006 ASCP annual meeting proceedings part I. J Clin Oncol. 2006;24(18S (June 20 Supplement)):6119a.

14. Webb T. Under-dosing study raises questions about ways to improve regimen adherence. J Natl Cancer Inst. 2004;96(16):1201-2.

15. Avorn J, Monette J, Lacour A, Bohn RL, Monane M, Mogun H, et al. Persistence of use of lipid-lowering medications: a cross-national study. JAMA. 1998;279(18):1458-62.

16. Wu EQ, Guerin A, Yu AP, Bollu VK, Guo A, Griffin JD. Retrospective real-world comparison of medical visits, costs, and adherence between nilotinib and dasatinib in chronic myeloid leukemia. Curr Med Res Opin. 2010;26(12): 2861-9.

17. Wood $L$. A review on adherence management in patients on oral cancer therapies. Eur J Oncol Nurs. 2012;16(4):432-8.

18. McCue DA, Lohr LK, Pick AM. Improving adherence to oral cancer therapy in clinical practice. Pharmacotherapy. 2014;34(5):481-94.

19. Verbrugghe $M$, Verhaeghe S, Lauwaert K, Beeckman D, Van Hecke A Determinants and associated factors influencing medication adherence and persistence to oral anticancer drugs: a systematic review. Cancer Treat Rev. 2013:39(6):610-21.

20. Andrade SE, Kahler KH, Frech F, Chan KA. Methods for evaluation of medication adherence and persistence using automated databases. Pharmacoepidemiol Drug Saf. 2006;15(8):565-74. discussion 575-567

21. Sikka R, Xia F, Aubert RE. Estimating medication persistency using administrative claims data. Am J Manag Care. 2005;11(7):449-57. 
22. Liu Y, Yang M, Chao J, Mulani PM. Greater refill adherence to adalimumab therapy for patients using specialty versus retail pharmacies. Adv Ther. 2010; 27(8):523-32.

23. Stockl KM, Shin JS, Lew HC, Zakharyan A, Harada AS, Solow BK, et al. Outcomes of a rheumatoid arthritis disease therapy management program focusing on medication adherence. J Manag Care Pharm. 2010;16(8):593604.

24. Waxman A, Chen SY, Boulanger L, Watson JA, Golden G. Factors associated with adherence to phosphodiesterase type 5 inhibitors for the treatment of pulmonary arterial hypertension. J Med Econ. 2013;16(2):298-306.

25. Tschida S, Aslam S, Khan TT, Sahli B, Shrank WH, Lal LS. Managing specialty medication services through a specialty pharmacy program: the case of oral renal transplant immunosuppressant medications. J Manag Care Pharm. 2013;19(1):26-41.

26. Moore S. Nonadherence in patients with breast cancer receiving oral therapies. Clin J Oncol Nurs. 2010;14(1):41-7.

27. St Charles M, Bollu V, Hornyak E, Coombs J, Blanchette C, De Angelo D. Predictors of treatment non-adherence in patients treated with imatinib mesylate for chonic myeloid leukemia. Poster II-186 at ASH 51st annual meeting. Blood. 2009;114:2209.

28. Hatoum HT, Lin SJ, Sasane M, Trent JC. Effectiveness of adjuvant imatinib in patients with gastrointestinal stromal tumor: results of a population-based, matched-cohort study. Curr Med Res Opin. 2012;28(5):805-14.

29. Henk HJ, Woloj M, Shapiro M, Whiteley J. Real-world analysis of tyrosine kinase inhibitor treatment patterns among patients with chronic myeloid leukemia in the United States. Clin Ther. 2015:37(1):124-33.

30. Winterhalder R, Hoesli P, Delmore G, Pederiva S, Bressoud A, Hermann F, et al., Group SI. Self-reported compliance with capecitabine: findings from a prospective cohort analysis. Oncology. 2011;80(1-2):29-33.

31. Wu EQ, Johnson S, Beaulieu N, Arana M, Bollu V, Guo A, et al. Healthcare resource utilization and costs associated with non-adherence to imatinib treatment in chronic myeloid leukemia patients. Curr Med Res Opin. 2010;26(1):61-9.

32. Barillet M, Prevost V, Joly F, Clarisse B. Oral antineoplastic agents: how do we care about adherence? Br J Clin Pharmacol. 2015;80(6):1289-302.

33. Walter T, Wang L, Chuk K, Ng P, Tannock IF, Krzyzanowska MK. Assessing adherence to oral chemotherapy using different measurement methods: lessons learned from capecitabine. J Oncol Pharm Pract. 2014;20(4):249-56.

34. Partridge $\mathrm{AH}$, Archer $\mathrm{L}$, Kornblith $\mathrm{AB}$, Gralow J, Grenier D, Perez E, et al. Adherence and persistence with oral adjuvant chemotherapy in older women with early-stage breast cancer in CALGB 49907: adherence companion study 60104. J Clin Oncol. 2010;28(14):2418-22.

35. Timmers L, Boons CC, Moes-Ten Hove J, Smit EF, van de Ven PM, Aerts JG, et al. Adherence, exposure and patients' experiences with the use of erlotinib in non-small cell lung cancer. J Cancer Res Clin Oncol. 2015:141(8):1481-91.

36. Eaddy MT, Cook CL, O'Day K, Burch SP, Cantrell CR. How patient costsharing trends affect adherence and outcomes: a literature review. Pharmacol Ther. 2012;37(1):45-55.

37. Hershman DL, Tsui J, Meyer J, Glied S, Hillyer GC, Wright JD, et al. The change from brand-name to generic aromatase inhibitors and hormone therapy adherence for early-stage breast cancer. J Natl Cancer Inst. 2014:106(11).

38. Neugut Al, Subar M, Wilde ET, Stratton S, Brouse CH, Hillyer GC, et al. Association between prescription co-payment amount and compliance with adjuvant hormonal therapy in women with early-stage breast cancer. J Clin Oncol. 2011:29(18):2534-42.

39. Streeter SB, Schwartzberg L, Husain N, Johnsrud M. Patient and plan characteristics affecting abandonment of oral oncolytic prescriptions. J Oncol Pract. 2011;7(3 Suppl):46s-51s.

40. Ganguli A, Clewell J, Shillington AC. The impact of patient support programs on adherence, clinical, humanistic, and economic patient outcomes: a targeted systematic review. Patient Prefer Adherence. 2016;10:711-25.

\section{Submit your next manuscript to BioMed Central and we will help you at every step:}

- We accept pre-submission inquiries

- Our selector tool helps you to find the most relevant journal

- We provide round the clock customer support

- Convenient online submission

- Thorough peer review

- Inclusion in PubMed and all major indexing services

- Maximum visibility for your research

Submit your manuscript at www.biomedcentral.com/submit 